\title{
Mastoid Emissary Vein Canal Incidence and Its Relationship with Jugular Bulb and Sigmoid Sulcus Anatomical Variations
}

\author{
Original Investigation \\ ๑ Rıdvan Pekçevik ${ }^{1}$, Aylin Öztürk ${ }^{2}$, Yeliz Pekçevik $^{2}$, $\odot$ Onur Toka ${ }^{3}$, \\ (-) Gülay Güçlü Aslann ${ }^{4}$ ๑ İbrahim Çukurova ${ }^{4}$ \\ ${ }^{1}$ Department of Radiology, İzmir Katip Çelebi University, Atatürk Training and Research Hospital, İzmir, Turkey \\ ${ }^{2}$ Department of Radiology, University of Health Sciences Turkey, Tepecik Training and Research Hospital, İzmir, \\ Turkey \\ ${ }^{3}$ Hacettepe University Faculty of Science, Department of Statistics, Ankara, Turkey \\ ${ }^{4}$ Department of Otorhinolaryngology, University of Health Sciences Turkey, Tepecik Training and Research Hospital, \\ İzmir, Turkey
}

\section{Abstract \\ ORCID ID of the authors: \\ R.P. 0000-0002-5706-5011; A.Ö. 0000-0001-9224-0252; Y.P. 0000-0003-1421-3376; O.T. $0000-0002-4025-4537$ G.G.A. 0000-0001-4021-4537; I.C. 0000-0002-2398-3391. \\ Cite this article as: Pekçevik R, Öztürk $A$, Pekçevik Y, Toka O, Güçlü Aslan G, Çukurova I. Mastoid Emissary Vein Canal Incidence and Its Relationship with Jugular Bulb and Sigmoid Sulcus Anatomical Variations. Turk Arch Otorhinolaryngol 2021; 59(4):244-52}

Corresponding Author: Rıdvan Pekçevik; ridvanpekcevik@gmail.com Received Date: 04.05 .2021 Accepted Date: 24.08 .2021

Content of this journal is licensed under a Creative Commons Attribution 4.0 International License. Available online at www.turkarchotolaryngol.net

\section{(c) (1) (\$)}

DOl: 10.4274/tao.2021.2021-4-26
Objective: We aimed to investigate the mastoid emissary vein (MEV) canal incidence and to identify its relationship with jugular bulb (JB) and sigmoid sulcus anatomical variations.

Methods: We retrospectively reviewed 1,300 patients with temporal bone computed tomography (CT) scans in January 2016 to March 2020. The presence and the diameter of the MEV canal, and the anatomical variations of the sigmoid sulcus and the JB were reviewed by two radiologists. High riding JB, JB diverticulum, dehiscent $\mathrm{JB}$, and anterior and lateral protrusion of the sigmoid sulcus were evaluated. All variables were summarized using descriptive statistics. The differences between the groups for categorical data were investigated using the chi-square test. Numeric variables were compared with the Mann-Whitney and the Kruskal-Wallis tests. Logistic regression models were constructed.

Results: The study included 1,269 patients of whom 694 were female (54.7\%) and 575 were male (45.3\%). Their mean age was $39.01 \pm 18.47$. Among them $915(72.1 \%)$ had the right and 871 (68.6\%) had the left MEV canal. Men were more likely to have the MEV canal on both sides. The presence of the right and left MEV canals was associated with the ipsilateral dominant JB/ sigmoid sulcus. The left $\mathrm{MEV}$ canal was associated with the left high riding JB and right dehiscent JB.

Conclusion: This is the largest patient population reported in the literature and allows a more precise estimate of the MEV canal incidence. We also classified the diameter of the MEV canal to identify clinically relevant, prominent MEV incidence. This is also the first study to demonstrate a relationship between the presence of the MEV canal, and the JB and sigmoid canal variations. Since both the prominent MEV and the JB variations may be symptomatic, knowing this association between them may have clinical relevance.

Keywords: Temporal bone, anatomy, emissary veins, jugular veins, jugular foramina, multidetector computed tomography, radiology 


\section{Introduction}

The sigmoid sinus begins at the junction of the transverse sinus and the superior petrosal sinus and runs inferiorly to communicate with the internal jugular vein (IJV). The jugular bulb (JB) located in the jugular fossa and connects to the IJV (1). Developmental abnormalities of the sigmoid canal and the JB have been proven to be clinically relevant, and may present with tinnitus, vertigo, and hearing loss (2).

While the IJV is the major extracranial venous drainage path, especially in prone position, the posterior fossa emissary veins are the primary route in upright position (3). The posterior fossa emissary veins connect the sigmoid sinus with the extracranial veins. The morphology of the posterior fossa dural sinuses, the emissary veins, and the JB are associated with the development of the brain, the shift to the postnatal type of circulation, and the postural hemodynamic change $(1,4)$. Regarding this close relationship, variations of the sigmoid sinus, the JB and the posterior fossa emissary veins may be associated.

A mastoid emissary vein (MEV) is a posterior fossa emissary vein that connects the sigmoid sinus and the posterior auricular or occipital vein crossing the mastoid foramen (4). Recognizing the MEV preoperatively is important. Because it may be a significant source of bleeding during skull base or middle ear surgery, detailed anatomical knowledge may prevent such complications (4). Air embolism may occur as a postoperative complication during a lateral suboccipital approach. Surgical materials used for hemostasis of the MEV may also cause complications (5).

The MEV may be the major drainage pathway to the extracranial venous system in the case of variations of dural sinuses or dural arteriovenous fistulas. It may also be used as an access route for the endovascular treatment of dural arteriovenous fistulas (6). The MEV may cause thrombosis of the sigmoid sinus after surgery of the middle ear. In addition, when the MEV is the major venous drainage of the posterior fossa, its ligation may result in venous ischemia and hemorrhage. Thrombosis of the MEV may occur as a complication of acute otomastoiditis. MEVs may also be a pathway for the spread of infections (7). Large MEVs may cause pulsatile tinnitus like JB and sigmoid sulcus variations (8).

MEVs can be evaluated with temporal bone computed tomography (CT) scan, CT angiography and magnetic resonance (MR) venography (8). Temporal CT scan and $\mathrm{CT}$ angiography are valuable tools for assessing the emissary veins and the venous vascular canals and are superior to $M R$ venography in depicting venous structures with slower flow and smaller diameters $(9,10)$.

We evaluated the MEV canal incidence in a large patient population and to investigate their relationship with JB and sigmoid sulcus anatomical variations using temporal bone CT scans.

\section{Methods}

\section{Patients}

This retrospective study was approved by the İzmir Health Sciences University, Tepecik Training and Research Hospital Ethics Committee (2020/14-66), and written informed consent was waived. In January 2016 to March 2020, we retrospectively reviewed 1,300 patients with temporal bone CT scans available at our department.

We excluded patients with previous mastoid, skull base, or posterior fossa surgery, dural sinus thrombosis or vascular malformations. Patients with poor technique or motion artifacts, and whose anatomy could not be properly assessed were excluded. We also excluded patients younger than five years old, since the JB may not be completely developed (10). Consequently, thirty-one patients were excluded, and 1,269 patients were included the study.

\section{CT Examination}

All temporal CT examinations were obtained using a 128-slice CT scanner (SOMATOM Definition AS, Siemens Healthcare, Erlangen, Germany) with a standard protocol without contrast administration. The scanning parameters included: detector collimation widths $128 \mathrm{x} 0.6$ $\mathrm{mm}$, tube voltage of $120 \mathrm{kV}$. Patients were scanned in the caudal to cranial direction with a scan revolution time of 1 second and pitch of 0.8 . Tube current was regulated by an automatic exposure control system (CARE Dose 4D; Siemens, Erlangen, Germany). Images were reconstructed in axial, coronal, and sagittal planes with a slice thickness of 1 $\mathrm{mm}$. The images were transmitted to the picture archiving and communication systems (PACS).

\section{Image Analysis}

The anatomical variations in the MEV, sigmoid sulcus, and JB were evaluated on $0.6 \mathrm{~mm}$ slice thickness images that were transmitted from PACS to the workstation (Aquarius Workstation; TeraRecon, San Mateo, California, USA). The images were evaluated on this workstation, in the axial, coronal, and sagittal planes by two radiologists, A.A. and R.P. (with 4 and 15 years of experience, respectively).

The MEV canal runs from the sigmoid sulcus extracranially, crossing the mastoid foramen. The presence of the MEV canal was noted and classified according to its size: less than $2 \mathrm{~mm}$, between 2 and $5 \mathrm{~mm}$, and more than $5 \mathrm{~mm}$ (Figure 1 ).

Developmental abnormalities of the JB (high riding JB, JB diverticulum, dehiscent JB), and variations of the sigmoid sulcus (anterior and lateral protrusion) were evaluated. 
When the roof of the JB was at the same level or above the floor of the internal acoustic canal, it was defined as high riding JB (Figure 2). A dehiscent JB was considered when the bony plate of the protruding JB toward the middle ear cavity was absent. Jugular bulb diverticulum was considered when there was an outpouching from the JB $(11,12)$ (Figure 3).

Sigmoid sulcus variation was characterized by an anterior and lateral position of the sigmoid sulcus in the mastoid bone $(10,13)$ (Figure 4). When the distance between the sigmoid sulcus lateral wall and the external tabula of the temporal bone was less than $2 \mathrm{~mm}$, and the distance between the sigmoid sulcus anterior wall and the external acoustic canal was less than $2 \mathrm{~mm}$, it was considered as a bulge of the sigmoid sulcus. The measurements were made on the axial images that showed the entire lateral semicircular canal.

Sigmoid sulcus/JB dominance was classified as right, left, or bilateral, according to the prominent vascular sulcus/bulb on one or both sides.

\section{Statistics}

IBM SPSS 23.0 statistical packages were used in statistical analysis. All variables were summarized with descriptive

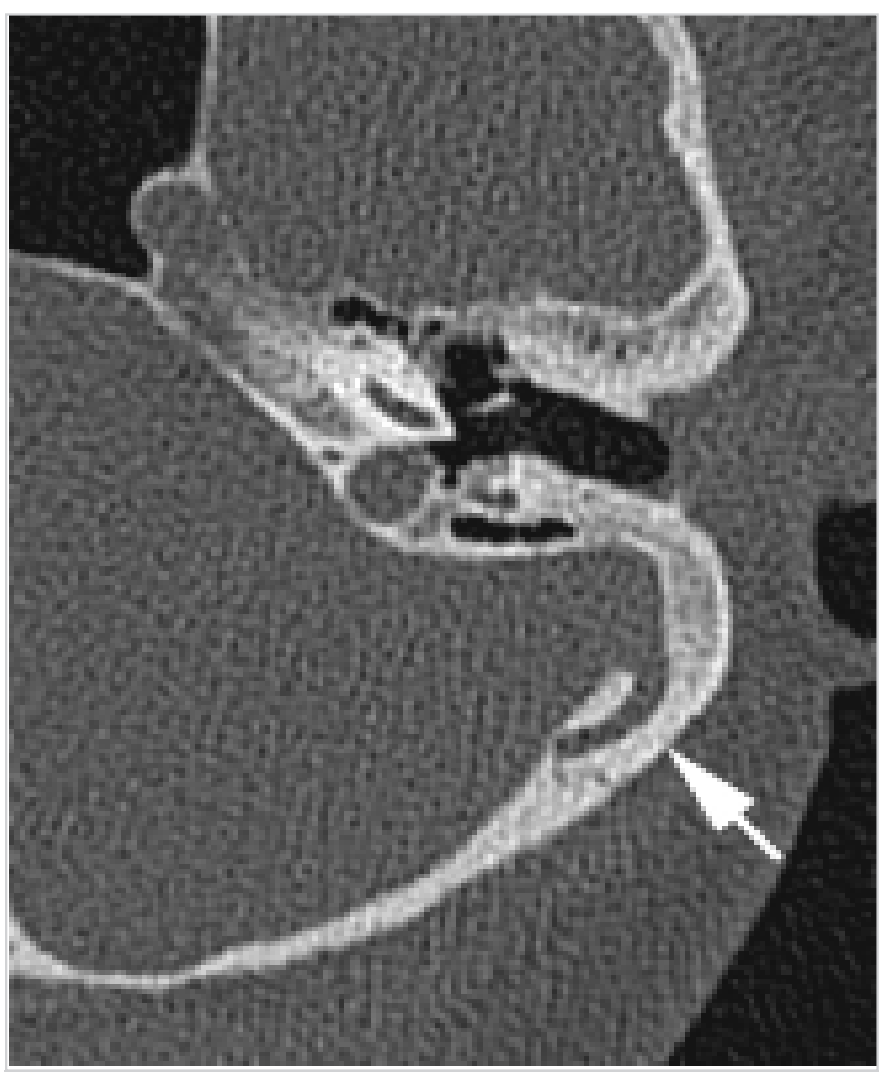

Figure 1. Temporal CT axial reformatted image shows the left mastoid emissary vein canal (arrow). The diameter of the canal is $2.5 \mathrm{~mm}$

CT: Computed tomography statistics. For some comparisons between different groups, the Kolmogorov-Smirnov Goodness-of-Fit test was used to investigate numerical variables to identify whether their distributions were parametric or non-parametric. The differences between groups for categorical data were investigated using the chi-square test, while the numerical variables between different groups were compared to each other with Mann-Whitney $U$ test in two labeled classes and the Kruskal-Wallis test in three or more labeled groups. Further statistical methods, logistic regression models were constructed for the right and the left MEV canals. The binary logistic regression models were explained and compared to each other with accuracy scores, odds ratios, and significances of some important independent variables. The models were also visualized using Receiver Operating Characteristic (ROC) graphics. $\mathrm{p}<0.05$ was considered

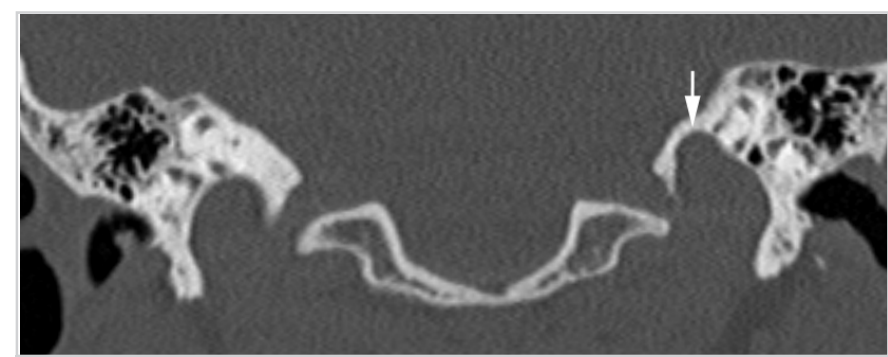

Figure 2. Temporal CT coronal reformatted image shows the left high riding jugular bulb (arrow)

CT: Computed tomography

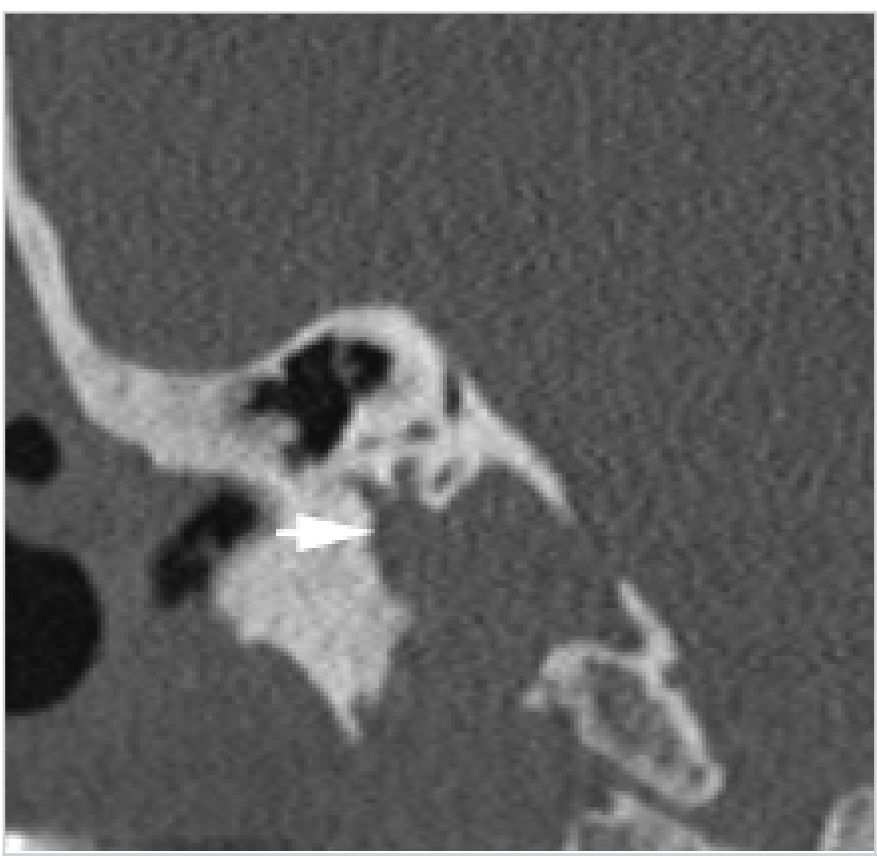

Figure 3. Temporal CT coronal reformatted image shows right high riding jugular bulb $(\mathrm{JB})$ and $\mathrm{JB}$ diverticulum, an extraluminal outpouching from the JB (arrow)

CT: Computed tomography 
statistically significant. An inter-observer reliability analysis with the kappa statistic was performed, and intraclass correlation coefficient (ICC) scores with 95\% CIs were calculated.

\section{Results}

After the initial screening, 1,269 patients, 694 females (54.7\%) and 575 males (45.3\%) with a mean age of $39.01 \pm 18.47$ were included in the study. Descriptive findings are given in Table 1 .

We did not have any patients with the MEV canal larger than $5 \mathrm{~mm}$. In our study population, 915 patients $(72.1 \%)$ had a right MEV canal, and 871 patients $(68.6 \%)$ had a

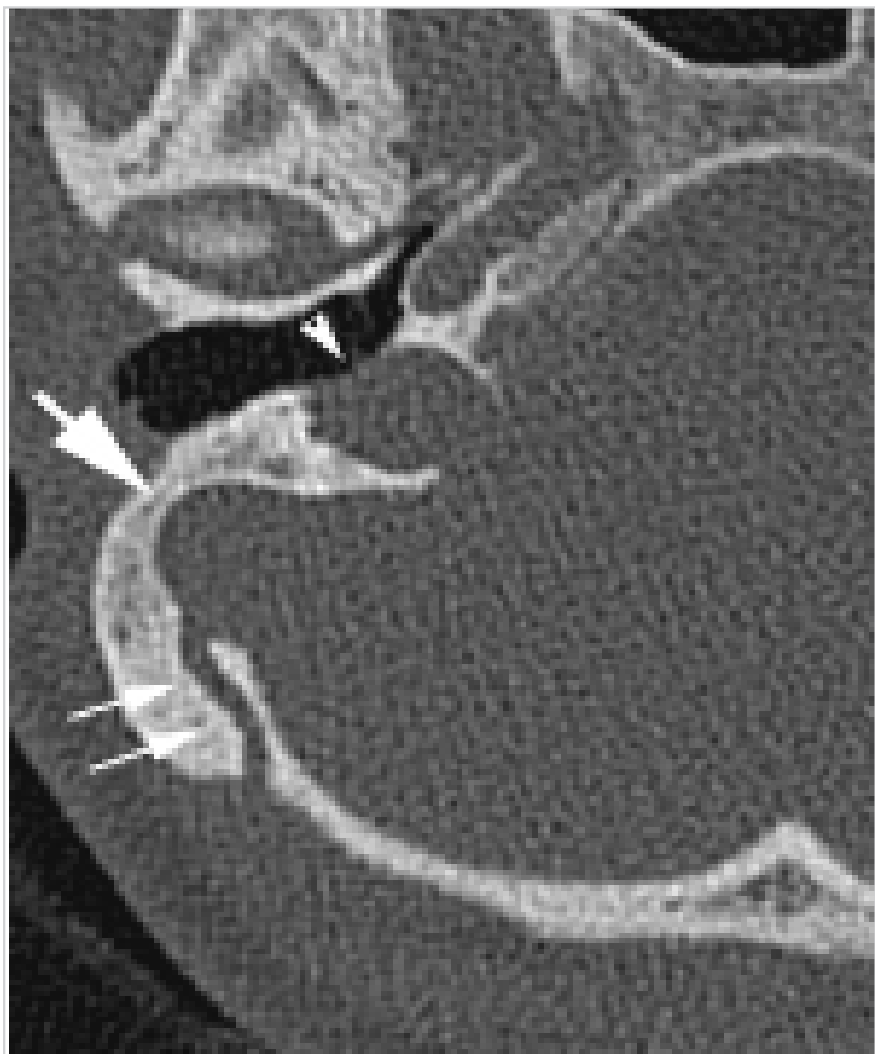

Figure 4. Temporal CT axial reformatted image shows lateral protrusion of the sigmoid sulcus into the mastoid bone (arrow). There is also a small $(<2 \mathrm{~mm})$ mastoid emissary vein canal (double arrow) and dehiscent jugular bulb (arrowhead)

CT: Computed tomography left MEV canal. Among all, 334 (26.3\%) of the right MEV canals and 291 (22.9\%) of the left MEV canals were larger than 2 millimeters.

Table 2 shows the mean age of the patients with right and left MEV canals. Comparison of patients by age showed no difference between the right $(\mathrm{p}=0.063)$ and the left $\mathrm{MEV}$ canals $(\mathrm{p}=0.367)$.

The frequency of the right and the left MEV canals in males and females are demonstrated in Table $3 . \mathrm{MEV}$ canals with a $2-5 \mathrm{~mm}$ diameter were less frequent in female patients ( $\mathrm{p}=0.002$ and $\mathrm{p}<0.001$, respectively).

The frequencies and percentages of the right and the left $\mathrm{MEV}$ canals by the existence of other variables are demonstrated in Tables 4 and 5. There was no relationship between the presence of a right $\mathrm{MEV}$ canal and JB or sigmoid sulcus

Table 1. The frequency of anatomical variations

\begin{tabular}{|c|c|c|c|}
\hline Variables & Labels & Patients (n) & $\%$ \\
\hline \multirow{2}{*}{ R MEV } & $<2 \mathrm{~mm}$ & 581 & 45.8 \\
\hline & $2-5 \mathrm{~mm}$ & 334 & 26.3 \\
\hline \multirow{2}{*}{ L MEV } & $<2 \mathrm{~mm}$ & 580 & 45.7 \\
\hline & $2-5 \mathrm{~mm}$ & 291 & 22.9 \\
\hline \multirow{2}{*}{ High riding JB } & $\mathrm{R}$ & 480 & 37.8 \\
\hline & $\mathrm{L}$ & 423 & 33.4 \\
\hline \multirow{2}{*}{ JB Diverticulum } & $\mathrm{R}$ & 134 & 10.6 \\
\hline & $\mathrm{L}$ & 108 & 8.5 \\
\hline \multirow{2}{*}{ Dehiscent JB } & $\mathrm{R}$ & 88 & 6.9 \\
\hline & $\mathbf{L}$ & 69 & 5.4 \\
\hline \multirow{2}{*}{$\mathrm{SS}$ variation } & $\mathrm{R}$ & 146 & 11.5 \\
\hline & $\mathrm{L}$ & 121 & 9.5 \\
\hline \multirow{3}{*}{ Venous dominance* } & $\mathrm{R}$ & 570 & 44.9 \\
\hline & $\mathbf{L}$ & 284 & 22.4 \\
\hline & $\mathrm{B}$ & 415 & 32.7 \\
\hline \multirow{3}{*}{ MEV dominance** } & $\mathrm{R}$ & 428 & 33.7 \\
\hline & $\mathrm{L}$ & 309 & 24.3 \\
\hline & B & 532 & 41.9 \\
\hline
\end{tabular}

R: Right, L: Left, B: Bilateral, MEV: Mastoid emissary vein, JB: Jugular bulb, SS: Sigmoid sulcus, "venous drainage dominance determined by the diameter of the SS and JB, ${ }^{* *}$ emissary vein dominance determined by the diameter of the MEV canal, $\mathrm{n}$ : Number

Table 2. Mean ages of patients with right and left MEV canals

\begin{tabular}{llll}
\hline & & Mean SD & p-value \\
\hline \multirow{2}{*}{ Right MEV } & $<2 \mathrm{~mm}$ & 37.2918 .90 & 0.063 \\
\cline { 2 - 4 } Left MEV & $2-5 \mathrm{~mm}$ & 39.7117 .46 & 38.9618 .82 \\
\end{tabular}


Table 3. Frequency distribution of MEV canals according to gender

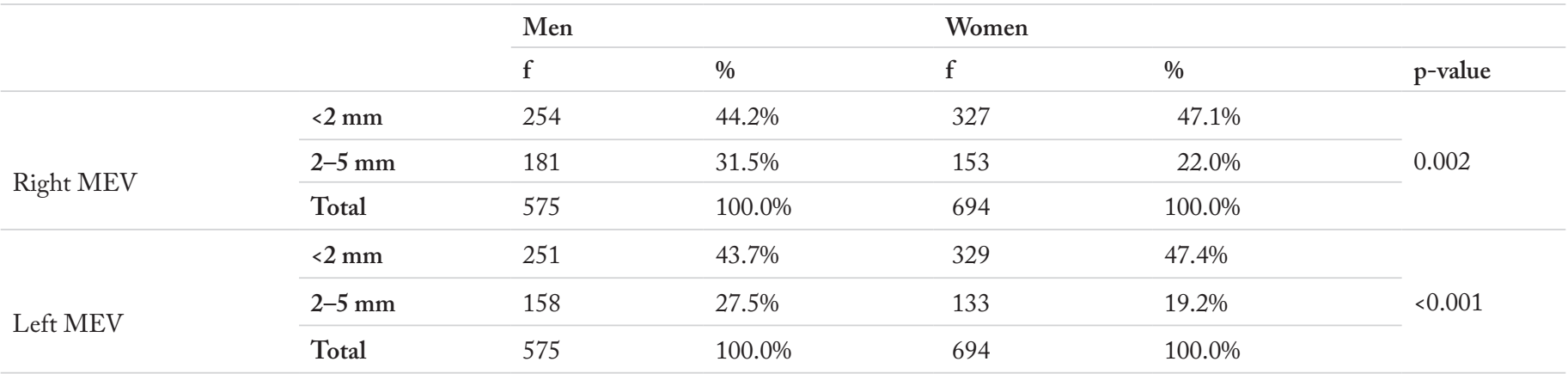

MEV: Mastoid emissary vein, f: frequency of MEV canals

Table 4. Frequencies and percentages of the right MEV according to the presence of JB and sigmoid sulcus variations

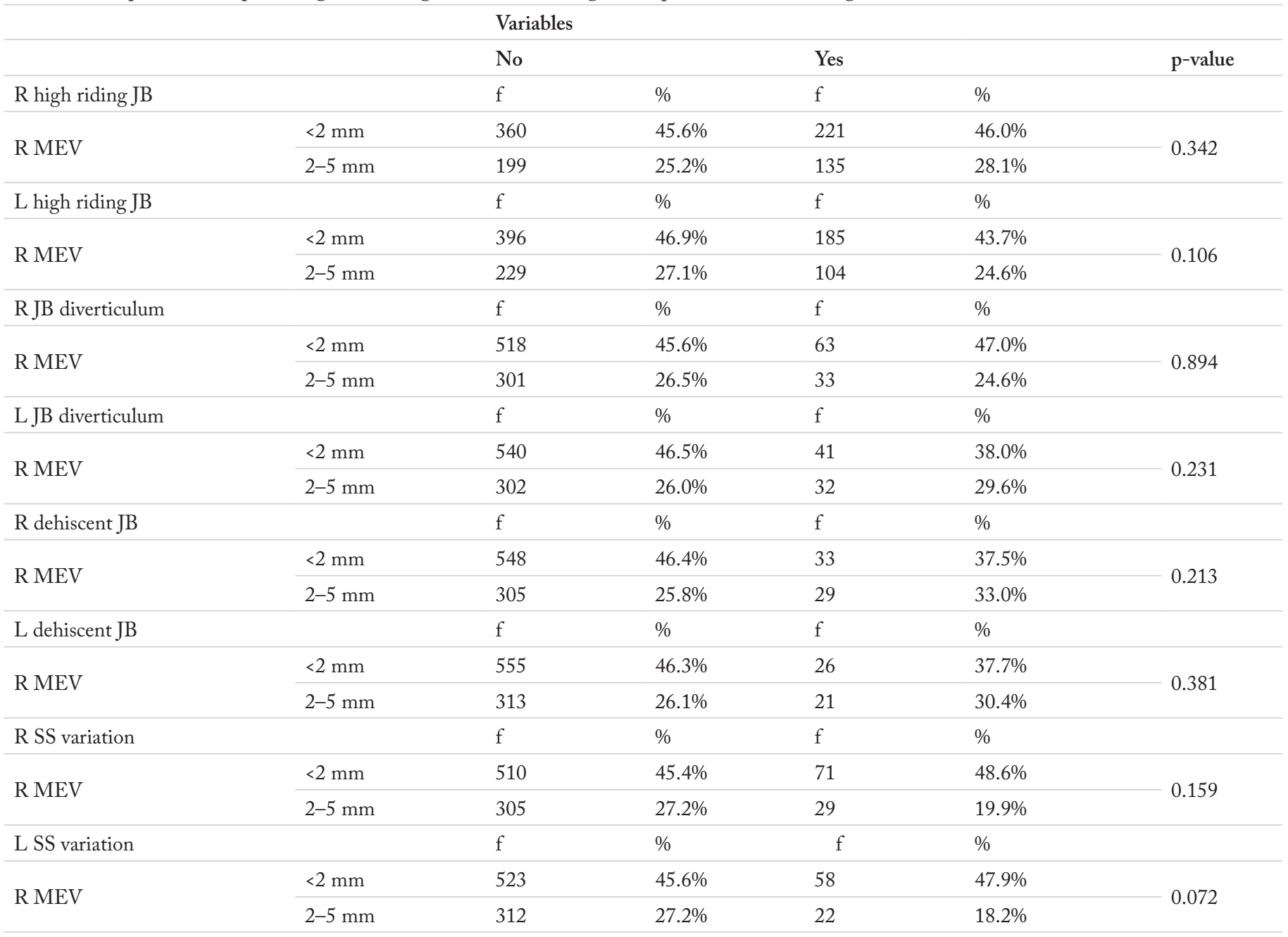

R: Right, L: Left, MEV: Mastoid emissary vein, JB: Jugular bulb, SS: Sigmoid sulcus, f: frequency of MEV canals

variations. A left MEV canal was associated with left high riding JB ( $\mathrm{p}=0.014)$ and right dehiscent JB $(\mathrm{p}=0.006)$.

The frequencies and percentages of right and left MEV canals by venous dominance, as determined by the sigmoid sulcus/JB diameter, are given in Table 6 . The presence of right and left MEV canals was associated with ipsilateral venous dominance. There was a relationship between a right dominant sigmoid sulcus/JB and the presence of a right MEV canal. A left dominant sigmoid sulcus/JB was associated with the presence of a left MEV canal. 
Table 5. Frequencies and percentages of the left MEV according to the presence of JB and sigmoid sulcus variations

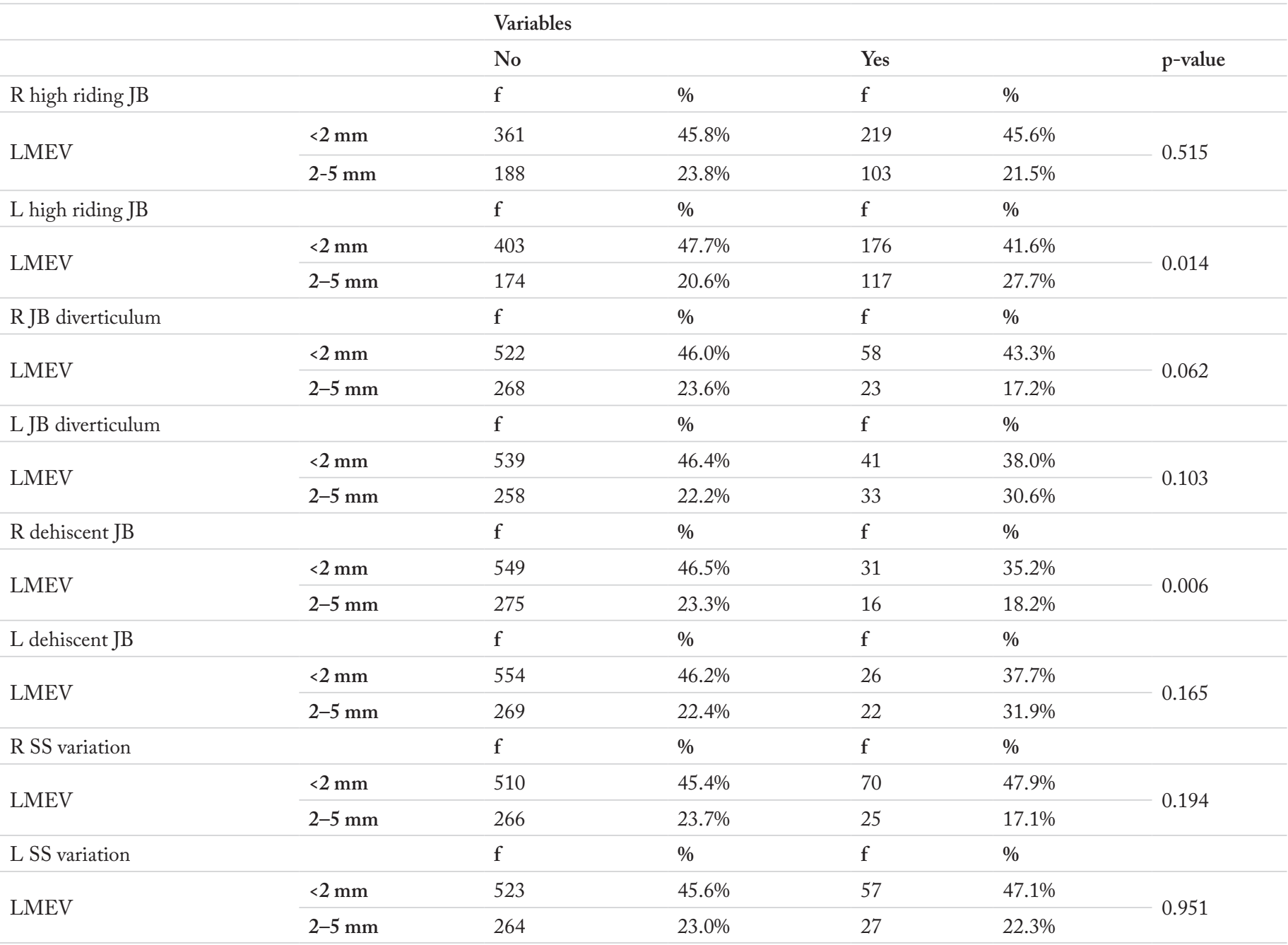

R: Right, L: Left, MEV: Mastoid emissary vein,JB: Jugular bulb, SS: Sigmoid sulcus, f: frequency of MEV canals

Table 6. Frequencies and percentages of right and left MEVs by venous dominance

\begin{tabular}{|c|c|c|c|c|c|c|c|c|}
\hline & & \multicolumn{6}{|c|}{ Venous dominance* } & \multirow{3}{*}{ p-value } \\
\hline & & \multicolumn{2}{|c|}{ Right } & \multicolumn{2}{|c|}{ Left } & \multicolumn{2}{|c|}{ Bilateral } & \\
\hline & & $\mathrm{f}$ & $\%$ & $\mathrm{f}$ & $\%$ & $\mathrm{f}$ & $\%$ & \\
\hline Right MEV & $2-5 \mathrm{~mm}$ & 163 & $28.6 \%$ & 55 & $19.4 \%$ & 116 & $28.0 \%$ & 0.003 \\
\hline Left MEV & $<2 \mathrm{~mm}$ & 276 & $48.4 \%$ & 112 & $39.4 \%$ & 192 & $46.3 \%$ & 0.003 \\
\hline
\end{tabular}

MEV: Mastoid emissary vein, *determined by the jugular bulb/fossa and sigmoid sulcus diameter, f: frequency of MEV canals

According to the logistic regression model, males were 1.3 times more likely to have a left MEV canal than females. Males were 1.88 times more likely to have a $2-5 \mathrm{~mm}$ left MEV canal than females. Further, patients with a left high riding JB were 1.59 times more likely to have a $2-5 \mathrm{~mm}$ left MEV canal.
Inter-observer agreement for the evaluation of the variations was excellent, with an ICC score ranging from 0.91 to 0.95 (95\% CI).

\section{Discussion}

We evaluated MEV canal incidences and JB and sigmoid sulcus variations in 1,269 patients. While the presence 
of a MEV canal was not found associated with age, males were more likely to have MEV on both sides in this large patient group. Regarding the closely related developmental factors between the dural sinuses and the emissary veins of the posterior fossa and the JB, we expected that the variations of the sigmoid sinus, the JB and the MEV could be associated (1). The presence of a right or a left MEV canal was associated with the prominent $\mathrm{JB}$ /sigmoid sulcus, ipsilaterally $(p=0.003)$. Although a right MEV canal was not related to JB or sigmoid sulcus variations, a left $\mathrm{MEV}$ was associated with a left high riding JB $(p=0.014)$ and a right dehiscent JB ( $\mathrm{p}=0.006)$. Patients with a left high riding JB were more likely to have a prominent left MEV in our study. Both the prominent MEV and the JB variations may be symptomatic and cause tinnitus as described by Kizildag et al. (10); thus, knowing this association may have clinical relevance.

Given its high spatial resolution, temporal bone CT scan is a valuable tool for assessing the temporal bone anatomy. It allows visualizing the fine anatomic structures of the middle and inner ears. The presence and variations of posterior fossa vascular canals can also be easily evaluated with temporal bone CT scans. With the introduction of multidetector techniques, images of less than $1 \mathrm{~mm}$ in diameter with multiplanar reformation improved diagnostic accuracy in the evaluation of the submillimetric structures (9). Brain $\mathrm{CT}$ angiography is also a valuable tool for assessing emissary veins. It allows to evaluate both emissary veins and dural venous sinuses (14). It is superior to MR venography in depicting venous structures with slower flow and smaller diameters (15). The major advantage of MR venography is lack of ionizing radiation.

In our study, the frequency of a right $\mathrm{MEV}$, both $<2 \mathrm{~mm}$ and $2-5 \mathrm{~mm}$, was slightly higher (72.1\%) than that of a left $\mathrm{MEV}$ (68.6\%). Frequency of MEV in cadaveric and imaging studies are reported within a wide range, mostly because of the number of patients and the methods used. Whether the MEV canal is more common on the right or on the left side is also controversial. A cadaveric study described a MEV in 63\% of 12 specimens (3). Louis et al. (5) reported a MEV prevalence of $98 \%$ on the right and of $72 \%$ on the left side in their cadaveric study of 200 specimens. Reis et al. (16) reported MEV in 89\% of their specimens. Koesling et al. (11) described the MEV canal incidence as $82 \%$ using high-resolution CT imaging. Pekcevik et al. (14), using CT angiography, identified MEV in $77.7 \%$ of their 166 patients, and more commonly on the left side. In their study using MR venography, Gulmez Cakmak et al. (15) found the MEV prevalence as $82.7 \%$ on the right side and as $81.4 \%$ on the left side in 247 patients. Tsutsumi et al. (17), using MRI, described the MEV incidence as $89.5 \%, 51.8 \%$ on the right and $24.7 \%$ on the left side. The relatively high incidence of right $\mathrm{MEV}$, in our study and most of the other studies in the literature, may be related to the similar mechanisms that are suggested to be the cause of the right JB and sigmoid sulcus dominance (1).

The major advantage of our study is that it is the largest patient population reported in the literature and allows a more precise estimate of the MEV canal incidence.

We also classified the MEV canals according to their diameter, especially to find the incidence of large-sized MEVs. There is no classification for the MEV canal in the literature. Previous studies only reported about the incidence of the MEV and did not classify these veins according to their diameter. We classified the MEV canal in our study, first to search for whether the relationship with JB and sigmoid sulcus variations is related to the diameter of the $\mathrm{MEV}$, and second to find the incidence of a large MEV canal. Recognizing prominent mastoid emissary veins may have clinical significance.

A large MEV may cause bleeding during the skull base or middle ear surgery (4). Air embolism or embolism of the surgical material that are used to achieve hemostasis of the MEV may occur (5). Emissary veins may be prominent in patients with high-flow vascular malformations and hypoplasia or aplasia of the jugular veins. A large MEV may be a potential target for cannulation during endovascular procedures involving the transverse or sigmoid sinus (6).

In our study, the MEV canal was accepted as prominent when larger than $2 \mathrm{~mm}$. We found the MEV canal prominent in 334 patients $(26.3 \%)$ on the right and 291 patients (22.9\%) on the left. Koesling et al. (11) described the MEV canal as prominent when larger than $1 \mathrm{~mm}$, and found this in $6 \%$ of the patients in their study. This is significantly lower compared to our study and may be due to either the CT image resolution or a lesser number of patients.

Enlarged posterior fossa emissary veins were found to be associated with craniofacial syndromes (18). Sarioglu et al. (19) found prominent MEV, larger than $2 \mathrm{~mm}$, more commonly in cochlear implant candidates (19.11\%) compared to the control group (6.3\%) in pediatric patients. Our study included both adult and pediatric patients older than 5 years old.

The presence of the MEV canal was not related to age in our study. Although the reason is not clear, males were more likely to have MEV on both sides than females in our large patient group. Louis et al. (5) found no relationship between gender or age. Gulmez Cakmak et al. (15) described the left MEV diameter higher in males, while Lang and Samii (20) observed that the MEV canal was less frequent in females. Dural venous sinus anomalies may also show gender differences like those in MEV. Hypoplasia of the left transverse sinus was reported to be more common in males (21). This may also give a clue about the relationship between 
the presence of the MEV and the JB and sigmoid sulcus variations.

The sigmoid sinus and jugular sinus, primitive JB, occur at a similar period in the fetus. The MEV vein originates from the ipsilateral sigmoid sinus and communicates with the extracranial veins $(1,4)$. Enlargement of the jugular sinus for becoming a JB is a dynamic process and not present at birth. It occurs after two years. Standing upright (rather than the horizontal position maintained in the in-utero and neonatal periods) causes an upward negative pressure generating from the heart to strike the jugular sinus at the jugular foramen. This results in bulbous enlargement of the primitive JB and sigmoid sinus. It is suggested that the relatively long left brachiocephalic vein may cause the dissipation of the energy transmitted to the left primitive JB. This may contribute to the higher incidence of large right JB and sigmoid sinus $(1,4)$. We identified an association between the dominant sigmoid sulcus/JB and the presence of the ipsilateral MEV. This may be due to the postural hemodynamic change that affects the morphology of the JB, dural sinuses and emissary veins of the posterior fossa at the same time.

Although we did not find any relationship between the presence of a right $\mathrm{MEV}$ and JB or sigmoid sulcus variations, a left MEV was associated with the existence of left high riding JB $(\mathrm{p}=0.014)$ and right dehiscent $\mathrm{JB}(\mathrm{p}=0.006)$. In our study, patients with left high riding JB were more likely to have a 2-5 $\mathrm{mm}$ left MEV. This relationship between a prominent left MEV and a high riding JB was not described previously. This is the first study to demonstrate the relationship between the presence of a MEV canal and JB and sigmoid canal variations. Both the prominent MEV and the high riding JB may be symptomatic and cause tinnitus (8). Knowing this relation may be clinically important. Gulmez Cakmak et al. (15) described an association between the presence of the MEV and variations of the transverse sinus, especially hypoplasia and aplasia of the transverse sinus, on the left side using MR venography. These findings are supported by the strong relationship between the embryological development of the posterior fossa emissary veins and dural venous sinuses (1). Although this study used MR venography to evaluate the MEV and dural venous sinuses, the findings support our hypothesis.

Our study also has some limitations. It is a retrospective study and we evaluated non-contrast CT images. The depiction of very small emissary veins, which is beyond the capability of the CT examinations, could have been missed. We evaluated the MEV in our study. Other posterior fossa emissary canals were not evaluated, since the MEV is considered as the most clinically important posterior fossa emissary vein and expected to show an association with the JB and sigmoid sulcus variations.
In conclusion, we evaluated the MEV canal incidence as well as JB and sigmoid sulcus variations. This is the largest patient population in the literature and allows for a more precise estimate of the MEV canal incidence. We also classified the diameter of the MEV canal to identify clinically relevant prominent MEV incidences. This is the first study to demonstrate the relationship between the presence of a MEV canal and JB and sigmoid canal variations. The presence of right and left MEV canals were associated with a prominent $\mathrm{JB} /$ sigmoid sulcus on the same side. A left MEV was associated with a left high riding JB and a right dehiscent JB. Patients with left high riding JB were more likely to have a prominent left MEV. Since both the prominent $\mathrm{MEV}$ and the JB variations may be symptomatic, knowing the incidence and association between the MEV canal and JB or sigmoid sulcus variations may have clinical relevance.

Ethics Committee Approval: This retrospective study was approved by the institutional review board of İzmir Health Sciences University, Tepecik Training and Research Hospital) (approval no: 2020/ 14-66, date: 23/12/2020).

Informed Consent: Written informed consent was waived.

Peer-review: Externally peer-reviewed.

\section{Authorship Contributions}

Conception: R.P., Y.P., A.Ö., G.G.A., İ.Ç., Design: R.P., Y.P., A.Ö., G.G.A., İ.Ç., Data Collection and/or Processing: R.P., Y.P., A.Ö., Analysis and/or Interpretation: R.P., Y.P., O.T., G.G.A., İ.Ç., Literature Review: R.P., Y.P., A.Ö., Writing: R.P., Y.P., Critical Review: R.P., Y.P., A.Ö., O.T., G.G.A., I.Ç.

Conflict of Interest: No conflict of interest was declared by the authors.

Financial Disclosure: The authors declared that this study received no financial support.

\section{Main Points}

- Developmental abnormalities of the sigmoid canal and jugular bulb (JB) have been proven to be clinically relevant, and can present with tinnitus, vertigo, and hearing loss.

- The mastoid emissary vein (MEV) is a posterior fossa emissary vein. It connects the sigmoid sinus and posterior auricular or occipital vein. Recognizing the MEV is clinically important.

- Presence of both right and left MEVs was associated with the ipsilateral prominent JB/sigmoid sulcus.

- The left MEV was associated with left high riding JB and right dehiscent JB. Patients with left high riding JB were more likely to have a prominent left MEV.

- Both prominent MEV and JB variations may be symptomatic and knowing this association may have clinical relevance. 


\section{References}

1. Okudera T, Huang YP, Ohta T, Yokota A, Nakamura Y, Maehara F, et al. Development of posterior fossa dural sinuses, emissary veins, and jugular bulb: morphological and radiologic study. AJNR AmJ Neuroradiol 1994; 15: 1871-83. [Crossref]

2. Friedmann DR, Le BT, Pramanik BK, Lalwani AK. Clinical spectrum of patients with erosion of the inner ear by jugular bulb abnormalities. Laryngoscope 2010; 120: 365-72. [Crossref]

3. San Millán Rúiz D, Gailloud P, Rüfenacht DA, Delavelle J, Henry $\mathrm{F}$, Fasel JH. The craniocervical venous system in relation to cerebral venous drainage. AJNR. Am J Neuroradiol 2002; 23: 1500-8. [Crossref]

4. Friedmann DR, Eubig J, McGill M, Babb JS, Pramanik BK, Lalwani AK. Development of the jugular bulb: a radiologic study. Otol Neurotol 2011; 32: 1389-95. [Crossref]

5. Louis RG Jr, Loukas M, Wartmann CT, Tubbs RS, Apaydin N, Gupta AA, et al. Clinical anatomy of the mastoid and occipital emissary veins in a large series. Surg Radiol Anat 2009; 31: 139-44. [Crossref]

6. Crocker M, Nesbitt A, Rich P, Bell B. Symptomatic venous sinus thrombosis following bone wax application to emissary veins. Br J Neurosurg 2008; 22: 798-800. [Crossref]

7. Rivet DJ, Goddard JK 3rd, Rich KM, Derdeyn CP. Percutaneous transvenous embolization of a dural arteriovenous fistula through a mastoid emissary vein. Technical note. J Neurosurg 2006; 105 : 636-9. [Crossref]

8. Pekçevik Y, Pekçevik R. Why should we report posterior fossa emissary veins? Diagn Interv Radiol 2014; 20: 78-81. [Crossref]

9. Lane JI, Lindell EP, Witte RJ, DeLone DR, Driscoll CL. Middle and inner ear: improved depiction with multiplanar reconstruction of volumetric CT data. Radiographics 2006; 26: 115-24. [Crossref]

10. Kizildag B, Bilal N, Yurttutan N, Sarica MA, Gungor G, Baykara $M$. The relationship between tinnitus and vascular anomalies on temporal bone CT scan: a retrospective case control study. Surg Radiol Anat 2016; 38: 835-41. [Crossref]
11. Koesling S, Kunkel P, Schul T. Vascular anomalies, sutures and small canals of the temporal bone on axial CT. Eur J Radiol 2005; 54: 335-43. [Crossref]

12. Park JJ, Shen A, Loberg C, Westhofen M. The relationship between jugular bulb position and jugular bulb related inner ear dehiscence: a retrospective analysis. Am J Otolaryngol 2015; 36: 347-51. [Crossref]

13. Eisenman DJ. Sinus wall reconstruction for sigmoid sinus diverticulum and dehiscence: a standardized surgical procedure for a range of radiographic findings. Otol Neurotol 2011; 32: 1116-9. [Crossref]

14. Pekcevik Y, Sahin H, Pekcevik R. Prevalence of clinically important posterior fossa emissary veins on CT angiography. J Neurosci Rural Pract 2014; 5: 135-8. [Crossref]

15. Gulmez Cakmak P,Ufuk F, Yagci AB, Sagtas E, Arslan M. Emissary veins prevalence and evaluation of the relationship between dural venous sinus anatomic variations with posterior fossa emissary veins: MR study. Radiol Med 2019; 124: 620-7. [Crossref]

16. Reis CV, Deshmukh V, Zabramski JM, Crusius M, Desmukh P, Spetzler RF, et al. Anatomy of the mastoid emissary vein and venous system of the posterior neck region: neurosurgical implications. Oper Neurosurg 2007; 61: 193-201. [Crossref]

17. Tsutsumi S, Ono H, Yasumoto Y. The mastoid emissary vein: an anatomic study with magnetic resonance imaging. Surg Radiol Anat 2017; 39: 351-6. [Crossref]

18. Jeevan DS, Anlsow P, Jayamohan J. Abnormal venous drainage in syndromic craniosynostosis and the role of CT venography. Childs Nerv Syst 2008; 24: 1413-20. [Crossref]

19. Sarioglu FC, Pekcevik Y, Guleryuz H, Olgun Y, Guneri EA. Variations of the vascular canals in the cochlear implant candidates. Int J Pediatr Otorhinolaryngol 2019; 123: 123-7. [Crossref]

20. Lang J Jr, Samii A. Retrosigmoidal approach to the posterior cranial fossa. An anatomical study. Acta Neurochir (Wien) 1991; 111: 147-53. [Crossref]

21. Goyal G, Singh R, Bansal N, Paliwal VK. Anatomical variations of cerebral MR venography: is gender matter? Neurointervention 2016; 11: 92-8. [Crossref] 